\title{
Light Enhancement in Surface-Enhanced Raman Scattering at Oblique Incidence
}

\author{
Sasani JAYAWARDHANA ${ }^{1}$, Lorenzo ROSA ${ }^{2}$, Ričardas BUIVIDAS $^{2}$, \\ Paul R. STODDART ${ }^{*}$, and Saulius JUODKAZIS ${ }^{2,3}$ \\ ${ }^{1}$ Centre for Atom Optics and Ultrafast Spectroscopy, Faculty of Engineering and Industrial Sciences, Swinburne \\ University of Technology, Hawthorn, VIC, 3122, Australia \\ ${ }^{2}$ Centre for Micro-Photonics, Faculty of Engineering and Industrial Sciences, Swinburne University of Technology, \\ Hawthorn, VIC 3122, Australia \\ ${ }^{3}$ Melbourne Centre for Nanofabrication, 151 Wellington Road, Clayton, VIC 3168, Australia \\ *Corresponding author: Paul R. STODDART $\quad$ E-mail: pstoddart@swin.edu.au
}

\begin{abstract}
Surface enhanced Raman scattering (SERS) measurements have been carried out at different focusing conditions using objective lenses of different numerical apertures. The experimentally observed dependence of SERS intensity of thiophenol-coated Ag nano-islands shows a close-to-linear scaling with the collection aperture. The linear relationship breaks down for large numerical apertures, which suggests that the scattering is anisotropic. Numerical simulations of realistically shaped Ag nano-islands were carried out, and the spatial distribution of hot-spots has been revealed at different heights near the nano-islands. Local field enhancements of up to 100 times were estimated. The simulation also suggests an explanation for the anisotropy in the scattering observed for larger numerical aperture objectives. This appears to be due to a reduction in the local field enhancement as the electric field vector component in the plane of the shallow metal islands reduces at larger angles of incidence.
\end{abstract}

Keywords: Surface-enhanced Raman scattering, metal island films, finite difference time domain

\section{Introduction}

Surface-enhanced Raman scattering (SERS) is a sensitive method for detecting analyte molecules in gas and liquid phases when they attach to or are present in the vicinity of the surface of plasmonic nanoparticles $[1,2]$. The particles act as nano-focusing lenses creating "hot-spots" in the electromagnetic field. Methods of controlling the shape, size, and ordering of nanoparticles, and their two- or three-dimensional (2D or 3D) arrangements provide a way to control the "hot-spots" [3-6]. Polarization and angular effects become important in the light field enhancement by plasmonic nano-focusing. When high-numerical aperture, $N A$, objective lenses are used to deliver excitation and to collect Raman scattering, the variation in polarization across the aperture and angular effects related to the Fresnel coefficient of reflection can become important.

Here we use microscope objectives with a range of numerical apertures to investigate how the light field enhancement and the resulting SERS signal depend on the angle of incidence. Numerical simulations have also been used to reveal how the hot-spots are distributed around the random pattern

Received: 16 June 2012 / Revised version: 20 June 2012

(C) The Author(s) 2012. This article is published with open access at Springerlink.com 
of self-organized islands of silver.

\section{Samples and methods}

Glass cover slips with a thickness of $100 \mu \mathrm{m}$ and refractive index $n$ of 1.47 were used without any surface treatment. A 2-nm layer of chromium (99.99\% ProSciTech) was first deposited under normal incidence for adhesion purposes. A nominal thickness of $100 \mathrm{~nm}$ of $\mathrm{Ag}$ (99.99\%, Sigma-Aldrich) was deposited on the cover slip under oblique angle deposition (OAD) in an Emitech K975X thermal evaporator [Fig. 1(a)]. During the deposition, the angle between the normal to the glass surface and the direction of the vapor flux $(\theta)$ was set to $86^{\circ}$. The deposition rate was maintained at $0.05 \mathrm{~nm} / \mathrm{s}$ with a chamber pressure of $1 \times 10^{-4} \mathrm{mbar}$, resulting in $\mathrm{Ag}$ nano-islands with a height of approximately $30 \mathrm{~nm}$. More details on sample preparation can be found elsewhere [7, 8]. The morphology of the $\mathrm{Ag}$ nano-islands was inspected by scanning electron microscopy [SEM, Fig. 1(b)].

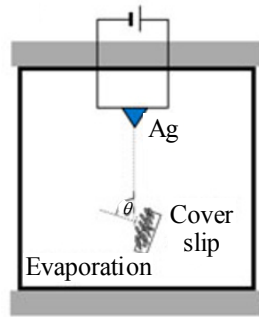

(a)

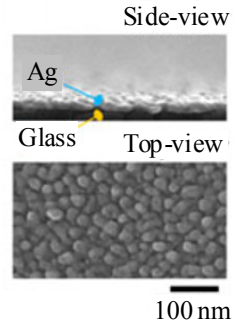

(b)
Fig. 1 Deposition of $\mathrm{Ag}$ island films: (a) evaporation of $\mathrm{Ag}$ nanoparticles on the flat glass cover slip where the vapor flux makes an oblique angle $\theta$ with respect to the surface normal and (b) SEM images showing a typical morphology of the resulting SERS substrates.

The surface of the nanoparticle film was then functionalized with thiophenol $\left(99 \%^{+}\right.$, Merck) by immersing in a 10-mM ethanolic solution for ten minutes, followed by rinsing in ethanol to remove any unbound molecules. Thiophenol was used as the test analyte as it is known to form a stable self-assembled monolayer (SAM) on Ag surfaces [9]. The thiophenol SERS spectra were acquired with an InVia Streamline microscope (Renishaw, UK) and 514.5-nm excitation in a backscattering geometry. Although the incident beam was linearly polarized, the polarization of the collected scattered light was not analyzed.

In order to compare SERS signals measured with different objective lenses, the data was normalized to represent about $1 \mathrm{~mW}$ at the sample. For comparison purposes, the baseline-to-peak heights of the four strongest thiophenol peaks $\left(1000 \mathrm{~cm}^{-1}\right.$, $1021 \mathrm{~cm}^{-1}, 1072 \mathrm{~cm}^{-1}$, and $1573 \mathrm{~cm}^{-1}$ ) were averaged to arrive at a single value for each spectrum. Table 1 shows the collection angles and numerical apertures of the objective lenses used in this work $(N A=$ $n \sin \alpha$, where $n=1$ for measurements in air).

Table 1 List of the objective lenses and corresponding numerical apertures NA used in this work.

\begin{tabular}{c|c|c|c}
\hline Number & Magnification & $\begin{array}{c}N A=n \sin \alpha \\
n=1 \text { in air }\end{array}$ & $\begin{array}{c}\text { Half-angle of focusing } \\
\alpha(\mathrm{deg})\end{array}$ \\
\hline 1 & $5 \times$ & 0.12 & $6.9^{\circ}$ \\
\hline 2 & $10 \times$ & 0.25 & $14.5^{\circ}$ \\
\hline 3 & $20 \times$ & 0.4 & $23.6^{\circ}$ \\
\hline 4 & $50 \times$ & 0.5 & $30^{\circ}$ \\
\hline 5 & $50 \times$ & 0.75 & $48.6^{\circ}$ \\
\hline 6 & $100 \times$ & 0.85 & $58.2^{\circ}$ \\
\hline
\end{tabular}

\section{Results and discussion}

\subsection{Optical characterization}

Figure 2 shows a typical SERS spectrum measured in back-reflection from a surface coated by Ag nano-islands and decorated by a thiophenol SAM. The four major SERS peaks were averaged for comparison of the focusing effects at different $N A$ values. To a first approximation, the average SERS signal closely follows a linear $\gamma=1$ dependence as shown in Fig. 3(a) but deviates at higher numerical apertures. The relationship between the solid angle of the collection cone and the numerical aperture is given in the Appendix. Panel (b) shows the intensity of the $521 \mathrm{~cm}^{-1}$ peak of $\mathrm{Si}$, which follows almost perfect linear scaling with 
the excitation/collection angle. Si is commonly used as a calibration standard and is understood to exhibit isotropic scattering. We therefore postulate that the small deviation from linearity in Fig. 3(b) is due to uncertainty in the specification of the objectives. In contrast, the non-linearity in the thiophenol signal is quite different to silicon and therefore indicates anisotropic scattering from the SERS substrate. This observation is in keeping with previous results from a variety of SERS substrates $[10,11]$.

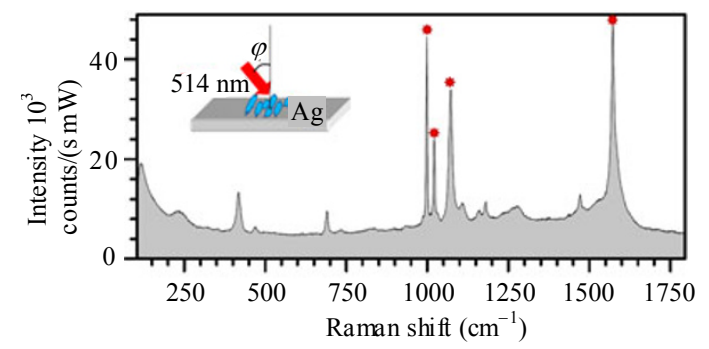

Fig. 2 Experimental SERS spectrum of thiophenol excited at an angle of incidence $\varphi=0$ degrees; focusing condition: $N A=$ 0.12 (the four Stokes Raman peaks used to calculate the average peak intensity are marked).

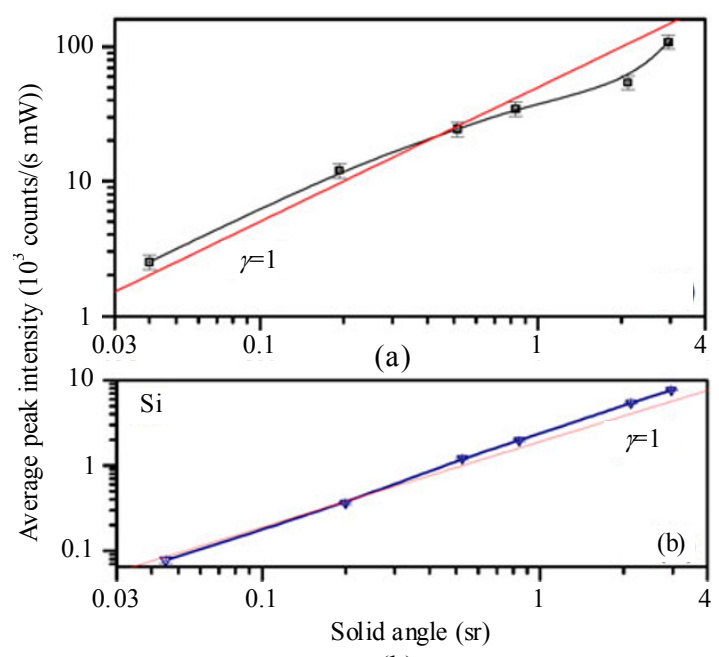

(b)

Fig. 3 Raman peak intensities as a function of solid angle: (a) the average thiophenol SERS peak intensity measured over different ranges of excitation-collection angles (the straight line shows a slope with $\gamma=1$ dependence) and (b) the intensity of the $521 \mathrm{~cm}^{-1} \mathrm{Si}$ Raman peak under the same range of focusing conditions (the solid angle is related to the half-angle of the focusing cone, $\alpha$, in Table 1 and was calculated according to the relationship given in the Appendix).

\subsection{Numerical simulation}

In order to examine the effect of the varying numerical aperture on the excitation capability given by the plasmonic field enhancement in the textured substrate, two 3D-finite difference time domain (FDTD) models have been compared through simulations performed using the FDTD solutions software (Lumerical Solutions, Inc.). The models, whose layout cross-sections are depicted in Figs. 4(a) and 4(c), are composed of a bulk glass domain with the refractive index $n=1.5$, on which $\mathrm{Ag}$ nanotextures are deposited in two different ways.

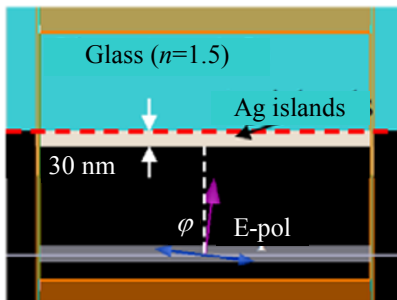

(a)

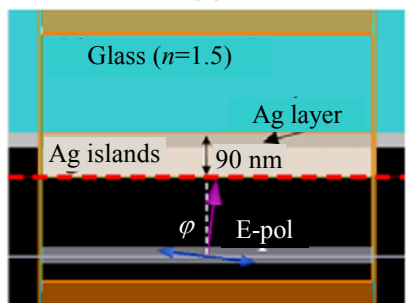

(c)

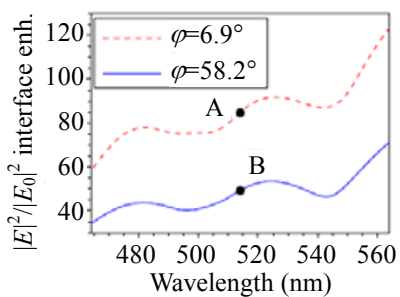

(b)

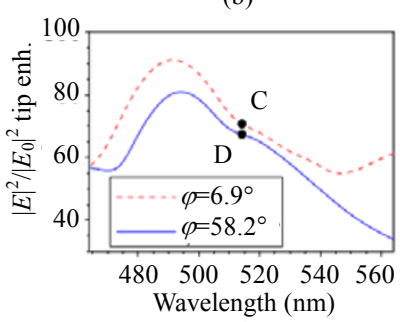

(d)
Fig. 4 3D-FDTD simulation layout: The $x z$-plane cross-section is shown with the glass domain $(n=1.5)$ covered by (a) a 30-nm Ag layer, which is patterned as Ag islands with the features of Fig. 1(b) and (c) a 60-nm patterned Ag layer on top of a 30-nm solid Ag layer. The plane wave source is tilted from the $z$-axis by an angle $\varphi$, which is compared with the marginal rays at focusing half-angle $\alpha$. The maximum simulated field enhancement $\left(|E|^{2} /\left|E_{0}\right|^{2}\right.$ for $\left.\left|E_{0}\right|^{2}=1\right)$ is shown in (b) for the Ag-glass interface as marked by the dashed line in panel (a), and (d) the Ag-air interface as marked by the dashed line in panel (c). $\varphi$ is equal to the maximum and minimum values of $\alpha$ according to Table 1 . The black dots (A-D) mark the cases examined in Fig. 6.

Firstly, in order to approximate the shape of the Ag islands in Fig. 1(b), the photo is numerically captured with six different levels of contrast, whose 
contours are assigned to six 5-nm Ag layers, as shown in Fig. 5. For the layout in Fig. 4(a), the islands are $30 \mathrm{~nm}$ high and are placed directly on the glass interface so that there is no direct electrical continuity, while in Fig.4(c), the islands are $60 \mathrm{~nm}$ high and are placed on top of a solid 30-nm Ag layer. This second structure is somewhat analogous to the Ag nanorod on the Ag film substrate fabricated by Liu et al. [11], also using oblique angle deposition. In both layouts, the structure is illuminated by an $x$-polarized linear plane-wave source, tilted by an angle $\varphi$ with respect to the $z$-axis, to highlight the contribution of waves at the beam boundary angle in each of the cases listed in Table 1.

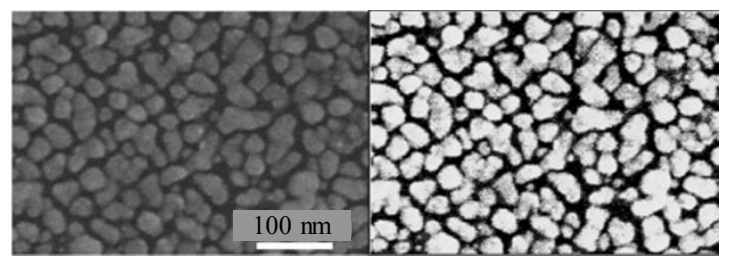

(a)

(b)

Fig. 5 Comparison between (a) SEM image from Fig. 1(b) and (b) captured image, where the six levels of grey correspond to Ag layer heights from 0 to $30 \mathrm{~nm}$.

The domain dimensions, given by the useable image size, are of $470 \mathrm{~nm} \times 325 \mathrm{~nm} \times 500 \mathrm{~nm}$, and a mesh size of $2 \mathrm{~nm}$ is used, which matches the granularity of the imported image. A single simulation takes about 3 hours to complete on a supercomputing Linux cluster, employing 8 nodes (each consisting of 8-core processors and 16-GB memory) and 10-GB total memory.

The simulations show a field enhancement of up to two orders of magnitude, but the hot-spot configuration is remarkably different: if there is no electrical continuity, the field is concentrated at the interface between the $\mathrm{Ag}$ islands and the glass substrate, while in the presence of the solid Ag layer, the maximum enhancement is found at the island tips. In the first case, Fig. 4(b) shows the maximum enhancement at the interface in a $100-\mathrm{nm}$ bandwidth centered at the laser wavelength of $514 \mathrm{~nm}$, while in the second case the tip enhancement over the same bandwidth is shown in Fig. 4(d). Only the two extreme values from Table 1 are shown for comparison. For the shorter island structure as it is captured from the image, the enhancement is generally increasing with the wavelength, while the enhancement of the taller islands peaks at around $490 \mathrm{~nm}$. This peak provides maximum enhancements in the bandwidth that are $20 \%$ to $50 \%$ larger than that at $514 \mathrm{~nm}$ and are relatively independent of the incidence angle.

The hot spot distribution has been investigated by taking the field cross-section on the $x y$-plane at the locations of the respective maximum fields, as shown in the log-scale plots of Fig. 6. The fields in Figs. 6(a) and 6(b) are taken at the Ag-glass interface and show a complementary enhancement distribution over the sample surface with respect to Figs. 6(c) and 6(d), taken at the Ag-air interface. The strongest contributions are given in specific hot-spots in constant positions within the image, but it is also possible to see a widely varying distribution of significant hot-spots all over the surface and their changing distribution for varying wave incident angle from $N A=0.12$ in Figs. 6(a) and 6(c) to $N A=0.85$ in Figs. 6(b) and 6(d).

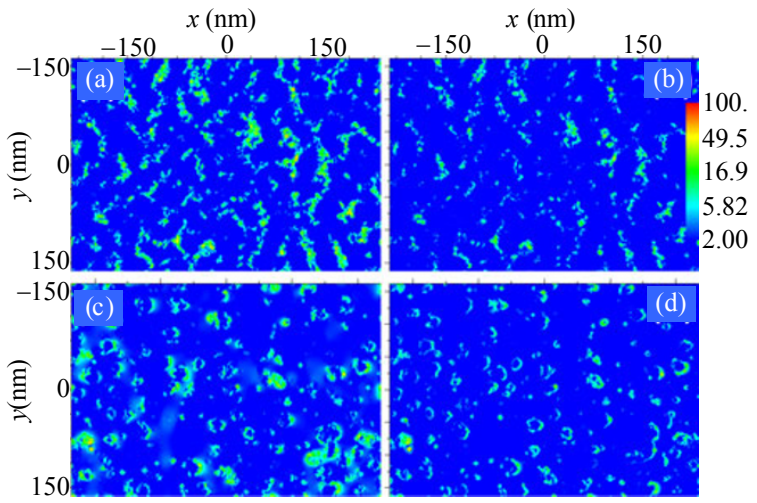

Fig. 6 Cross-sectional plots in the $x y$-plane of the field enhancement $|E|^{2}$ for 514-nm wavelength at the base of the 30-nm Ag islands for (a) $N A=0.12\left(|E|_{\text {max }}^{2}=85\right)$ and (b) $N A=$ $0.85\left(|E|_{\text {max }}^{2}=50\right)$; at the tip of the 60 -nm islands for (c) $N A=$ $0.12\left(|E|_{\text {max }}^{2}=70\right)$ and (d) $N A=0.85\left(|E|_{\text {max }}^{2}=67\right)$ (the plots are in logarithmic scale). 
The excitation efficiency tends to decrease for wider incident angles, but this effect is more pronounced for the nano-island film than for the taller rods on the Ag film. The decrease is to be expected for large angles of incidence as the electric field component in the $x y$-plane is reduced and becomes less effective in exciting dipole resonances between neighbouring structures. In this interpretation, dipole resonances in the thin metal island film tend to be confined to the $x y$-plane, whereas the thicker nanorod on the Ag film structure can excite dipoles over a larger range of angles extending out of the plane. Extending this idea, the high $N A$ objectives add more $k$-vectors to the incident beam, which could potentially activate a larger number of hot-spots, particularly in more extended 3D structures. The metal-island surface may also become more reflective at larger angles of incidence, which may lead to a reduction in enhancement. These concepts must be confirmed in future work.

Despite a decrease in the excitation efficiency for marginal rays, the higher- $N A$ objectives are able to collect more light from the sample. This causes a more even sample illumination and is useful in situations where photo-bleaching is present. Moreover, with a more regularly textured surface, the effect can be used to tailor the activation of specific parts of the sample with nanometric precision.

\section{Conclusions}

A self-assembled monolayer of thiophenol was detected on the surface of silver nano-islands at different excitation-collection angles. A close-to-linear SERS intensity scaling was observed, with a small but significant decrease in the measured intensity for the highest numerical aperture objective lenses of $N A=0.75$ and 0.85 . This indicates that the scattering from the $\mathrm{Ag}$ island films is anisotropic. Numerical simulations revealed the locations of hot-spots at the interface between the substrate and nano-islands of $\mathrm{Ag}$, or on the island tips if placed on a conducting base layer of Ag. The model suggests that intensity enhancement of up to 100 times can be achieved, and the enhancement in hot-spot areas was predicted to decrease at larger incident angles (larger $N A$ ), particularly for the thin nano-islands. We postulate that this reduction in the hot spot intensity accounts for the anisotropy in the scattering observed with large $N A$ objectives.

\section{Appendix}

The relationship between the solid angle $\Omega=$ $2 \pi(1-\cos \alpha)[\mathrm{sr}]$ and numerical aperture of the objective lens $N A=n \sin \alpha$ is given below. In this work, $\alpha$ is the half-angle of the focusing cone with apex $\alpha$, and $n$ is the refractive index of the medium in which focusing occurs $(n=1$ for air in our experiments). By squaring and summation, one finds

$$
\frac{\Omega^{2}}{4 \pi^{2}}-\frac{\Omega}{\pi}+\frac{N A^{2}}{n^{2}}=0
$$

with roots

$$
\Omega=2 \pi\left(1 \pm \sqrt{1-(N A / n)^{2}}\right)
$$

Taking a series expansion for $\left(1-(N A / n)^{2}\right)^{1 / 2}$ in the case where $N A / n<1$, this reduces to

$$
\Omega=\pi\left[\left(\frac{N A}{n}\right)^{2}+\frac{(N A / n)^{4}}{4}+\frac{(N A / n)^{6}}{8}+\ldots\right] .
$$

In general, the approximation $\Omega \approx \pi N A^{2} / n^{2}$ is only valid for the case where $N A / n<<1$.

\section{Acknowledgment}

This work was supported by the Australian Research Council under Discovery Project grant DP1092955 and Lumerical program acquired via DP120102980. The authors thank Dr. Sharath Sriram (RMIT) for assisting with the SEM analysis.

Open Access This article is distributed under the terms of the Creative Commons Attribution License which permits any use, distribution, and reproduction in any 
medium, provided the original author(s) and source are credited.

\section{References}

[1] W. Smith, "Practical understanding and use of surface enhanced Raman scattering/surface enhanced resonance Raman scattering in chemical and biological analysis," Chemical Society Reviews, vol. 37, no. 5, pp. 955-964, 2008.

[2] K. Hering, D. Cialla, K. Ackermann, T. Dorfer, R. Moller, H. Schneidewind, et al., "SERS: a versatile tool in chemical and biochemical diagnostics," Analytical and Bioanalytical Chemitry, vol. 390, no. 1, pp. 113-124, 2008.

[3] Y. Nishijima, L. Rosa, and S. Juodkazis, "Surface plasmon resonances in periodic and random patterns of gold nano-disks for broadband light harvesting," Optics Express, vol. 20, no. 10, pp. 11466-11477, 2012.

[4] Y. Yokota, K. Ueno, S. Juodkazis, V. Mizeikis, N. Murazawa, H. Misawa, et al., "Nano-textured metallic surfaces for optical sensing and detection applications," Journal of Photochemistry. Photobiology A: Chemistry, vol. 207, no. 1, pp. 126-134, 2009.

[5] R. Brown and M. Milton, "Nanostructures and nanostructured substrates for surface-enhanced
Raman scattering (SERS)," Journal of Raman Spectroscopy, vol. 39, no. 10, pp. 1313-1326, 2008.

[6] M. J. Banholzer, J. E. Millstone, L. Qin, and C. A. Mirkin, "Rationally designed nanostructures for surface-enhanced Raman spectroscopy," Chemical Society Reviews, vol. 37, no. 5, pp. 885-897, 2008.

[7] S. Jayawardhana, G. Kostovski, A. P. Mazzolini, and P. R. Stoddart, "Optical fiber sensor based on oblique angle deposition," Applied Optics, vol. 50, no. 2, pp. 155-162, 2011.

[8] J. D. Driskell, S. Shanmukh, Y. Liu, S. B. Chaney, X. J. Tang, Y. P. Zhao, et al., "The use of aligned silver nanorod arrays prepared by oblique angle deposition as surface enhanced Raman scattering substrates," Journal Physical Chemistry C, vol. 112, no. 4, pp. 895-901, 2008.

[9] M. A. Bryant and J. E. Pemberton, "Surface Raman-scattering of self-assembled monolayers formed from 1-alkanethiols at Ag," Journal American Chemical Society, vol. 113, no. 10, pp. 3629-3637, 1991.

[10] B. Pettinger, U. Wenning, and H. Wetzel, "Angular resolved Raman-spectra from pyridine adsorbed on silver electrodes," Chemical Physics Letters, vol. 67, no. 1, pp. 192-196, 1979.

[11] Y. J. Liu, J. G. Fan, Y. P. Zhao, S. Shanmukh, and R. A. Dluhy, "Angle dependent surface enhanced Raman scattering obtained from a Ag nanorod array substrate," Applied Physics Letters, vol. 89, no. 17, pp. 173134, 2006. 